\title{
AVALIAÇÃO DO COMPORTAMENTO DE ESPÉCIES DE Eucalyptus spp. NA ZONA DA MATA PERNAMBUCANA. I: RESULTADOS DO PRIMEIRO ANO - 2001'
}

João Luis Barboza Coutinho², Venézio Felipe dos Santos ${ }^{3}$, Rinaldo Luiz Caraciolo Ferreira ${ }^{4}$ e José Carlos Barboza Nascimento ${ }^{5}$

\begin{abstract}
RESUMO - O trabalho objetivou selecionar, para a região da Zona da Mata de Pernambuco, cultivares de Eucalyptus e, ou, identificar clones com alto potencial de produtividade. As espécies Eucalyptus saligna Sm., E. dunnii Maiden, E. benthamii Maiden \& Cambage, E. tereticornis Sm., E. urophylla S.T. Blake, E. pilularis Sm., E. camaldulensis Dehnh., E. robusta Sm., E. grandis W. Hill ex Maiden e E. citriodora Hook estão sendo avaliadas por sete anos, observando-se as seguintes variáveis: sobrevivência (\%), bifurcação (\%), altura (m) e diâmetro $(\mathrm{cm})$. O delineamento utilizado foi em blocos ao acaso, com quatro repetições, sendo a parcela constituída de 35 plantas. Aos 12 meses, as espécies apresentaram sobrevivência superior a 95,0\%, não foi registrado bifurcação na espécie E. dunnii.e E. citriodora exibiu o valor máximo de $35,0 \%$, enquanto nas demais espécies os valores foram inferiores a $15,0 \%$. Os melhores desempenhos de altura e diâmetro ocorreram em E. citriodora, E. urophylla, E. camaldulensis, E. saligna, E. grandis e E. dunnii.
\end{abstract}

Palavras-chave: Eucaliptos, sobrevivência, altura e diâmetro.

\section{PERFORMANCE OF Eucalyptus spp. SEEDLINGS IN THE "DRY RAINFOREST" REGION OF THE STATE OF PERNAMBUCO, BRAZIL. I: RESULTS OF THE FIRST YEAR.}

\begin{abstract}
Due to its high rainfall and predominance of hot climate conditions all over the year, the 'dry rainfall' region of the State of Pernambuco, located in the Northeastern part of Brazil, appears to be suitable to the growth and development of many forest tree species such as those of the genus Eucalyptus. In this trial, seedlings of 10 Eucalyptus species were tried, namely: Eucalyptus saligna Sm., E. dunnii Maiden, E. benthamii Maiden \& Cambage, E. tereticornis Sm., E. urophylla S.T. Blake, E. pilularis Sm., E. camaldulensis Dehnh., E. robusta Sm., E. grandis W. Hill ex Maiden and E. citriodora Hook. Observations and measurements will be carried out during seven years, taking into account mainly: survival (\%), plant height and crown width. A complete randomised block experimental design was used with four replicates, with 35 plants per plot. At the first evaluation, held 12 months after planting, the following data were obtained: the majority of Eucalyptus species showed a survival rate higher than 95,0\%; in relation to forked trees, only $\boldsymbol{E}$. dunnii, has not presented such characteristic, while E. citriodora. showed the highest rate (35\%) and the others, values below 15\%; highest plant height and diameter at breast heigth (DBH) were found among seedlings of E. citriodora, E. urophylla, E. camaldulensis, E. saligna E. grandis and E. dunnii.
\end{abstract}

Key words:Woody species, varieties, survival, forked trees, forest.

\footnotetext{
${ }^{1}$ Recebido em 14.11.2002 e aceito para publicação em 10.08.2004.

${ }^{2}$ IPA. E-mail: <jluis@ipa.br $>$.

${ }^{3}$ Pesquisador IPA. E-mail: <venezio@ipa.br>.

${ }^{4}$ Departamento de Engenharia Florestal - UFRPE. E-mail: <rmsantos@elogica.com.br>.

${ }^{5}$ Assistente-técnico IPA.
} 


\section{INTRODUÇÃO}

A família Myrtaceae, à qual pertence o gênero Eucalyptus, tem como seu centro de origem o continente australiano. Este gênero possui cerca de 600 espécies e variedades endêmicas, ocorrendo ainda na Indonésia e em ilhas adjacentes (PRYOR, 1976).

No Brasil, o Eucalyptus foi introduzido por D. Pedro I, em 1825, que plantou mudas das espécies robusta e tereticornis no Jardim Botânico do Rio de Janeiro (MOURA et al., 1980).

Atualmente, a área de florestas plantadas no Brasil atinge cerca de 4,6 milhões de hectares em regime de produção, dos quais 2,5 milhões são de eucaliptos (GALVÃO, 2000).

As mesorregiões Metropolitana do Recife e Mata pernambucana distinguem-se das demais regiões do estado, principalmente pelas suas condições edafoclimáticas, vocacionadas naturalmente para exploração de espécies arbóreas. Apresentam índices pluviométricos, balanço hídrico, umidade e temperatura bastante favoráveis ao desenvolvimento de plantios florestais de múltiplo uso com as espécies do gênero Eucalyptus. Outro fator preponderante para o desenvolvimento de projetos florestais nessa região é, sem dúvida, o consumo de biomassas para fins energéticos, que na região atinge um volume de 4,28 milhões de metros cúbicos por ano, sendo utilizados $33,3 \%$ em domicílios e 41,3\% para fins industriais e residenciais (CAMPELLO et al., 1999). Na composição da matriz energética de Pernambuco no ano 2000, estimada em 6.454.000 toneladas de energéticos totais (1.000 tEP), o grupo biomassa participou com $39,7 \%$, sendo $14,5 \%$ de origem florestal (lenha e carvão) e $25,2 \%$ de origem da cana-de-açúcar (álcool e bagaço de cana) (ESTUDO..., 1999). Diante da crescente demanda de madeira para a produção de energia, considerando a impossibilidade de exploração da mata Atlântica, que atualmente ocupa uma área de aproximadamente 0,42\% de sua cobertura original (SILVA FILHO et al., 1998), sendo alvo de permanente vigilância e preservação, transfere-se à região semi-árida, ecossistema caatinga e brejos de altitude, a responsabilidade de atender a essa demanda.

A Zona da Mata de Pernambuco, formada pela mesorregião metropolitana de Recife e pela Mata pernambucana, é onde está inserida a agroindústria sucroalcooleira, que compreende uma área de 1.015,03 mil hectares (ESTUDO..., 1999), dos quais apenas 840 mil apresentam aptidão climática para a exploração da cana-de-açúcar. Dessa área, $45 \%$ apresenta declividade superior a $20^{\circ}$ (IBGE, 1996), o que torna a atividade nessas áreas de baixo ou nulo retorno econômico. Essas áreas marginais para a cana-de-açúcar podem ser viabilizadas economicamente com a exploração de projetos florestais para múltiplos usos com eucalipto. Dessa forma, este trabalho objetivou selecionar espécies de eucaliptos para a Zona da Mata de Pernambuco.

\section{MATERIAL E MÉTODO}

Com o objetivo de avaliar 10 espécies de Eucalyptus spp., a Empresa Pernambucana de Pesquisa Agropecuária (IPA) instalou um experimento na Estação Experimental de Itapirema, Goiana,PE. A área experimental apresenta as seguintes características: latitude de $07^{0} 34^{\prime} 00^{\prime \prime} \mathrm{S}$, longitude de $35^{\circ} 00^{\prime} 00^{\prime} \mathrm{WGr}$, tipo climático úmido megatérmico, com o período de chuvas concentrado de março a julho, precipitação pluviométrica média de $1.979,1 \mathrm{~mm}$, temperatura média de $25,0^{\circ} \mathrm{C}$, umidade relativa média de $65 \%$ e altitude de $14 \mathrm{~m}$. O solo, classificado como Podzólico Vermelho-Amarelo, topografia plana, apresentou na camada arável as seguintes características: $2 \mathrm{mg} / \mathrm{dm}^{3} \mathrm{P}$ e 0,$03 ; 0,05 ; 1,00$; e $0,55 \mathrm{cmolc} / \mathrm{dm}^{3} \mathrm{de} \mathrm{K}$, $\mathrm{Al}, \mathrm{Ca}$ e $\mathrm{Mg}$, respectivamente. Mediante os resultados, foi aplicada 1,0 t/ha de calcário dolomítico em 21.02.2000. A adubação básica foi constituída de uma mistura na cova $(20 \mathrm{~cm} \times 20 \mathrm{~cm} \times 30 \mathrm{~cm})$ de $3 \mathrm{~L}$ de torta de filtro de usina de açúcar e $36 \mathrm{~g}$ de $\mathrm{P}_{2} \mathrm{O}_{5}$. O experimento foi instalado no dia 28.04.2000, e, posteriormente, foram realizadas duas adubações de cobertura, aos 30 dias, com $25 \mathrm{~g}$ de $\mathrm{N}$ e $10 \mathrm{~g}$ de $\mathrm{K}_{2} \mathrm{O}$ e, aos 60 dias, com 25 $\mathrm{g}$ de $\mathrm{N}$ e $15 \mathrm{~g}$ de $\mathrm{K}_{2} 0$.

As mudas de eucaliptos foram produzidas em telados na própria estação experimental, oriundas de sementes com ampla diversidade genética, cedidas pela Embrapa Florestas. As espécies avaliadas foram: Eucalyptus saligna Smith, E. dunnii Maiden, E. benthamii Maiden et Cambage, E. tereticornis Smith, E. urophylla S. T. Blake, E. pilularis Smith, E. camaldulensis Dehnh, E. robusta Smith, E. grandis W. Hill ex Maiden e E. citriodora Hook.

Diante da diversidade de procedências das espécies, foram identificadas plantas por tratamento e parcela, visando à seleção daquelas que apresentaram maior adaptabilidade às condições agroclimáti- 
cas da região. Segundo Burley e Wood (1976), o crescimento em altura e a sobrevivência das plantas são as características mais importantes para a avaliação da adaptação de uma espécie. Andrew e Wright (1976) relacionaram como características de importância econômica a altura, o diâmetro e a sobrevivência, sugerindo que elas devam ser consideradas para fins de avaliação final. Já Smith (1962) afirmou que a altura, em virtude de estar menos sujeita a variações ambientais, é uma característica mais precisa do que o diâmetro na seleção de espécies. Golfari (1972) relatou que a altura é o parâmetro mais importante para a sobrevivência das árvores em competição, sendo um dos índices mais seguros para determinar se a espécie foi plantada em local apropriado.

O delineamento experimental adotado foi de blocos ao acaso, com 10 tratamentos e quatro repetições. A parcela experimental foi constituída de uma área de $21 \mathrm{~m} \mathrm{x} 10 \mathrm{~m}$, com um total de 35 plantas, no espaçamento de $3 \mathrm{~m}$ x $2 \mathrm{~m}$, sendo as mensurações realizadas em 15 plantas, na área útil de $90 \mathrm{~m}^{2}$. As médias foram comparadas pelo teste de Tukey a $5 \%$ de probabilidade.
O experimento será avaliado anualmente por um período de sete anos, observando-se os seguintes parâmetros: sobrevivência (\%), bifurcação (\%), altura $(\mathrm{m})$ e diâmetro à altura do peito (DAP, cm); por ocasião da colheita será avaliado o volume cúbico com casca $\operatorname{VCCC}\left(\mathrm{m}^{3} /\right.$ árvore) e o incremento médio anual com casca IMACC ( $\mathrm{m}^{3} /$ árvore).

\section{RESULTADOS E DISCUSSÃO}

A precipitação pluviométrica ocorrida nos 12 primeiros meses foi de $2.398,80 \mathrm{~mm}$. Na Tabela 1 são apresentadas as procedências e os resultados de sobrevivência (\%), bifurcação (\%), altura (m) e o DAP $(\mathrm{cm})$, nas 10 espécies de eucaliptos avaliadas.

As sementes de E. pilularis apresentaram germinação e vigor baixos; no campo foram necessários vários replantios, o que possivelmente, nesse primeiro ano, tenha comprometido sua "performance" fenológica (Tabela 1).

Com relação a sobrevivência no primeiro ano, apenas as espécies E. tereticornis, E. benthamii e E. pilularis apresentaram níveis iguais a 98,3\%; 96,9\% e 95,0\%, respectivamente, enquanto as demais apresentaram $100 \%$ de sobrevivência.

Tabela 1 - Procedência, porcentagem de sobrevivência e bifurcação, altura e diâmetro à altura do peito (DAP) de 10 espécies de Eucalyptus spp. aos 12 meses de idade

Table 1 - Provenance, survival percentage and branching, height and width of 10 especies of Eucalyptus ssp, at 12 months of age

\begin{tabular}{|c|c|c|c|c|c|}
\hline Espécie & Procedência & $\begin{array}{c}\text { Sobrevivência } \\
(\%)\end{array}$ & $\begin{array}{c}\text { Bifurcação } \\
(\%)\end{array}$ & $\begin{array}{c}\text { Altura } \\
(\mathrm{m})\end{array}$ & $\begin{array}{l}\text { DAP } \\
(\mathrm{cm})\end{array}$ \\
\hline E. benthamii & Colombo, PR ex-Wentworth Falls, NSW & 96,9 & 1,96 & $4,71 \mathrm{c}$ & $4,84 \mathrm{a}$ \\
\hline E. camaldulensis & $\begin{array}{l}\text { Brasilândia MG, Terrana (Austrália); } \\
\text { Morada Nova, MG; Petford (QLD) }\end{array}$ & 100,0 & 10,00 & $5,97 \mathrm{ab}$ & $5,63 \mathrm{a}$ \\
\hline E. citriodora & ACESITA; Terrara (Austrália) & 100,0 & 35,00 & $6,35 \mathrm{a}$ & $5,55 \mathrm{a}$ \\
\hline E.dunnii & Colombo, PR & 100,0 & 0,00 & $5,53 \mathrm{abc}$ & $5,76 \mathrm{a}$ \\
\hline E. grandis & $\begin{array}{l}\text { Coff"s Harbour; Aguaí, SP; } \\
\text { África do Sul }\end{array}$ & 100,0 & 8,33 & $5,85 \mathrm{abc}$ & $5,44 \mathrm{a}$ \\
\hline E. pilularis & $\begin{array}{l}\text { CAF MR ex-Gallangowan State } \\
\text { Forest, QLD }\end{array}$ & 95,0 & 5,26 & $3,47 \mathrm{~d}$ & $3,36 \mathrm{~b}$ \\
\hline E.robusta & $\begin{array}{l}\text { Itapetininga SP; Ouro Fino, MG; } \\
\text { Guaíba, RS }\end{array}$ & 100,0 & 10,00 & $5,17 \mathrm{bc}$ & $5,07 \mathrm{a}$ \\
\hline E. saligna & Itatinga, SP; Capão Bonito, SP & 100,0 & 6,66 & $5,89 \mathrm{ab}$ & $5,33 \mathrm{a}$ \\
\hline E. Tereticornis & Brasilândia, MG; Avaré, SP & 98,3 & 11,86 & $5,32 \mathrm{abc}$ & $5,22 \mathrm{a}$ \\
\hline E. urophylla & $\begin{array}{l}\text { Camzaquã, SP; Itatinga, SP; } \\
\text { Brasilândia, MG; Anhembi, SP; } \\
\text { Guaíba, RS }\end{array}$ & 100,0 & 15,00 & $6,32 \mathrm{a}$ & $5,79 \mathrm{a}$ \\
\hline \multirow{2}{*}{\multicolumn{4}{|c|}{$\begin{array}{l}\text { Coeficiente de variação experimental } \\
\text { Coeficiente de variação entre plantas dentro da parcela }\end{array}$}} & $6,99 \%$ & $9,76 \%$ \\
\hline & & & & $18,96 \%$ & $26,96 \%$ \\
\hline
\end{tabular}

Nas colunas, as médias seguidas das mesmas letras não diferem enter si, pelo teste de Tukey $(P<0,05)$. 
A espécie E. dunnii não apresentou bifurcação e, a espécie E. citriodora apresentou o percentual máximo de $35 \%$. As demais espécies apresentaram um nível de bifurcação de até $15 \%$.

As cinco espécies com as melhores "performances" em altura foram: E. citriodora $(6,35 \mathrm{~m})$, E. urophylla $(6,32 \mathrm{~m})$, E. camaldulensis $(5,97 \mathrm{~m})$, E. saligna $(5,89$ $\mathrm{m})$ e $E$. grandis $(5,85 \mathrm{~m})$. Observou-se que apenas $E$. pilularis, com a altura de $3,47 \mathrm{~m}$ (menor), diferiu das demais espécies, pelo teste de Tukey $(\mathrm{P}<0,05)$.

Com relação ao DAP, as cinco melhores marcas foram para: E. urophylla $(5,79 \mathrm{~cm}), E$. dunnii $(5,76 \mathrm{~cm})$, E. camaldulensis $(5,63 \mathrm{~cm})$, E. citriodora $(5,55 \mathrm{~cm})$ e E. saligna $(5,33 \mathrm{~cm})$. Apenas a espécie E. pilularis diferiu estatisticamente das demais, pelo mesmo teste e nível de significância.

Os resultados de altura obtidos neste trabalho nas espécies E. citriodora, E. urophylla, E. camaldulensis, E. grandis e E. tereticornis foram superiores aos conseguidos por Drumond et al. (1998) nessas mesmas espécies, aos 12 meses de idade, em tabuleiros costeiros do Estado de Sergipe: 17,6\%, 24,6\%, $6,6 \%, 36,0 \%$ e $18,8 \%$, respectivamente. Com relação ao DAP das referidas espécies, os resultados foram também superiores: $23,3 \%, 28,7 \%, 19,8 \%, 32,7 \%$ e $30,5 \%$, respectivamente. Ainda, comparando os resultados desses mesmos autores, aos 30 meses de idade, constatouse que a espécie E. tereticornis, neste trabalho, apresentou uma superioridade em altura de 6,6\%, enquanto nas demais espécies se observou uma variação para menor de aproximadamente $5 \%$.

$\mathrm{Na}$ avaliação de desempenho de E. camaldulensis e E. urophylla em altura, Souza e Carvalho (1984), em Teresina, PI, aos 36 meses de idade, constataram que essas espécies apresentaram melhor resultado com relação à altura, 65,1 e 21,8\%, e DAP, 41,0 e 8,46\%, respectivamente, comparativamente às registradas neste trabalho.

Em Petrolina, PE, Souza et al. (1985) avaliaram o comportamento das espécies $E$. camaldulensis, $E$. grandis e E. urophylla aos 6, 12 e 24 meses de idade, cujos resultados foram inferiores aos obtidos neste trabalho em $82,6 \%, 103,8 \%$ e $151,8 \%$, respectivamente, com essas espécies nas mesmas condições.

A avaliação de comportamento das espécies $E$. camaldulensis e E. urophylla aos 36 meses de idade realizada por Lima et al. (1985), em Souza, PB, apre- sentaram resultados superiores aos deste trabalho, para altura, em $61,5 \%$ e $10,1 \%$, respectivamente, e para DAP em 45,5\%; em E. camaldulensis e E. urophylla, o resultado obtido neste trabalho foi superior $10,1 \%$ ao obtido por esses autores.

Testes de procedências de eucaliptos realizados por Gurgel Filho et al. (1978) no município de MogiGuaçu, SP, aos 24 meses de idade, das espécies $E$. camaldulensis, E. grandis, E. pilulares e E. tereticornis, apresentaram resultados superiores aos obtidos neste estudo, com relação à altura, em 29,5\%,53,5\%, 58,5\% e $11,3 \%$, respectivamente, e ao DAP, em 14,3\%, 22,2\%, $63,7 \%$ e $4,4 \%$, respectivamente. Com E. tereticornis foram conseguidos resultados superiores aos obtidos pelos referidos autores.

As plantas selecionadas mediante a sua maior "performance" de altura foram de E. saligna, com 16 plantas com a altura entre 8,0 e $6,0 \mathrm{~m} ;$ E. dunnii, 14 plantas com a altura ente $6,5 \mathrm{~m} \mathrm{e} 7,0 \mathrm{~m} ; E$. benthamii, 08 plantas com a altura entre $4,9 \mathrm{~m} \mathrm{e} 6,6 \mathrm{~m}$; E. tereticornis, 15 plantas com a altura entre $6,0 \mathrm{~m} \mathrm{e} \mathrm{7,1} \mathrm{m;} \mathrm{E.} \mathrm{urophylla,}$ 26 plantas com altura entre 7,0 e 8,0 m; E. pilularis, seis plantas com altura entre 5,0 e 6,8 m; E. camaldulensis, 12 plantas com altura entre 6,8 e 7,0 m; E. robusta, 14 plantas com altura entre 6,8 e 7,0 m; E. grandis, 18 plantas com altura entre 6,0 e $8,0 \mathrm{~m}$; e E. citriodora, 23 plantas com altura entre 6,0 e 8,0 m.

\section{CONCLUSÕES}

As espécies E. citriodora, E. urophylla, E. camaldulensis, E. saligna, E. grandis e E. dunnii apresentaram as melhores "performances" de altura e diâmetro.

Os resultados de primeiro ano, ainda que incipientes, têm alimentado uma perspectiva de se obter, no final do trabalho, uma seleção de espécies de $E u$ calyptus aptas às características agroclimáticas da região da Mata Pernambucana.

\section{AGRADECIMENTOS}

Os autores agradecem ao Ministério da Integração Nacional (MI), sucessor da Superintendência do Desenvolvimento do Nordeste (SUDENE), e à Embrapa Florestas, na pessoa do Dr. Jarbas Yukio Shimizu, e, ainda, aos concluintes do Curso de Engenharia Florestal/2001 da Universidade Federal Rrural de Pernambuco o apoio. 


\section{REFERÊNCIAS BIBLIOGRÁFICAS}

ANDREW, I. A.; WRIGHT, H. L. Assessment and analisys. In: BURLEY, J.; WRIGHT, H. L. A manual on species and provenances research with particular reference to the tropics. Oxford: Commonwealth Forestry Institute, 1976. p. 108-30.

BURley, J.; WOOD, P. J. A manual on species and provenances research with particular reference to the tropics. Oxford: Commonwealth Forestry Institute, 1976. 226p.

CAMPELLO, F. B. et al. Diagnóstico florestal da Região Nordeste. Brasília: IBAMA, 1999. 15p. (IBAMA. Boletim Técnico, 2).

DRUMOND, M. A.; OLIVEIRA, V. R.; CARVALHO, O. M. Comportamento silvicultural de espécies e procedências de Eucalyptus na região dos tabuleiros costeiros do Estado de Sergipe.

Revista Árvore, v.22, n. 1, p.133-142, 1998.

ESTUDO de viabilidade de desenvolvimento de projetos florestais de múltiplos usos no Brasil; cenário atual e tendências do mercado doméstico e internacional de produtos florestais. São Paulo: JPEngenharia/WI/BNDES/PNUD, 1999. v.2, 105p. (Projeto PNUD/BRA//97/043).

GALVÃO, A. P. M. Reflorestamento de propriedade rurais para fins produtivos e ambientais: um guia para ações municipais e regionais. Brasília: EMBRAPA-CTT, 2000. 351p.

GOLFARI, L. $2^{\circ}$ Curso de zoneamento florestal: A introdução das espécies exóticas e o processo de adaptação. Belo Horizonte: [s. n.], 1972. 12p.
GURGEL FILHO, O. A. et al. Testes de procedências de Eucalyptus e Pinus spp no Estado de São Paulo. São Paulo: Instituto Florestal, 1978. 40p. (IF. Boletim Técnico, 28).

LIMA, P. C. F.; SOUZA, S. M.; BEZERRA, A. N. Comportamento de Eucalyptus camaldulensis Dehnh aos 36 meses de idade em Souza, PB. Petrolina: EMBRAPACPTASA, 1985. 3p.(EMBRAPA-CPATSA, Pesquisa em Andamento, 37).

MOURA, V. P. G. et al. Avaliação de espécies de Eucalyptus em Minas Gerais e Espírito Santo: resultados parciais. Brasília: EMBRAPA-CPAC, 1980. 104p. (EMBRAPA-CPAC. Boletim de Pesquisa, 1).

PRYOR, L. Biology of Eucalyptus. London: Edward Arnold, 1976. 82p.

SILVA FILHO, A. A. et al. Mapeamento da cobertura florestal nativa lenhosa do Estado de Pernambuco. Recife: PNUD/ FAO/IBAMA, 1998. 32p. (PNUD/FAO/IBAMA. Documento de Campo FAO, 17).

SOUZA, S. M.; CARVALHO, J. H.

Comportamento de Eucalyptus

camaldulensis Dehnh aos 36 meses de idade em Teresina, PI. Petrolina: EMBRAPA-CPATSA, 1984. 3p. (EMBRAPACPATSA. Pesquisa em Andamento, 26).

SOUZA, S. M.; LIMA, P. C. F.; PIRES, I. E. Comportamento de Eucalyptus camaldulensis Dehnh em Pernambuco,PE, aos 36 meses de idade. Petrolina: EMBRAPACPATSA, 1985. 5p. (EMBRAPA-CPATSA. Pesquisa em Andamento, 32).

SMITH, D. M. The practice of siviculture. 7. ed. New York: John Wiley \& Sons, 1962. 578p. 